\title{
Visual OntoBridge: Semi-automatic Semantic Annotation Software
}

\author{
Miha Grcar and Dunja Mladenic \\ Jozef Stefan Institute, Dept. of Knowledge Technologies, Jamova cesta 39, \\ 1000 Ljubljana, Slovenia \\ \{Miha.Grcar, Dunja.Mladenic\}@ijs.si
}

\begin{abstract}
Machine learning methods have been successfully used for data labeling, also referred to as data annotation, either in semi-automatic or fully automatic way. We present a system for semi-automatic annotation of Web service schemas and other resources with the motivation to support efficient browse and search through those resources and to enable efficient composition and execution of Web services. The presented system, Visual OntoBridge (VOB), provides a graphical user interface and employs a set of machine learning algorithms to support the user in the annotation task.
\end{abstract}

Keywords: annotation, ontologies, graphs, machine learning, text mining, PageRank, Visual OntoBridge.

\section{Introduction and Motivation}

In order to support efficient browse and search through resources and to enable efficient composition and execution of Web services, these resources need to be semantically annotated. In our work, the semantic annotation is defined as a set of interlinked domain-ontology instances being associated with the resource being annotated. For example, let us assume that our resource is a database table. We want to annotate its fields in order to provide compatibility with databases from other systems. Further on, let us assume that this table has a field called "employee_name" that contains employee names (as given in Fig. 1, left side). On the other hand, we have a domain ontology containing knowledge and vocabulary about companies (an excerpt is given in Fig. 1, right side). In order to state that our table field in fact contains employee names, we first create an instance of the domain-ontology concept Name and associate it with the field. We then create an instance of Person and link it to the instance of Name via the hasName relation, and an instance of Company and link it to the instance of Person via the hasEmployee relation. Such annotation (shown in the middle in Fig. 1) indeed holds the desired semantics: the annotated field contains names of people which some company employs (i.e. names of employees). Formulating annotations in one of the ontology-description languages (e.g. Web Service Modeling Language <http://www.wsmo.org/wsml/>) is not a trivial task and requires specific expertise. Therefore, the users would benefit from a system that allows them to visualize the resource and the domain ontology (much like this is done in Fig. 1), create instances by clicking on concepts, and interlink them by 


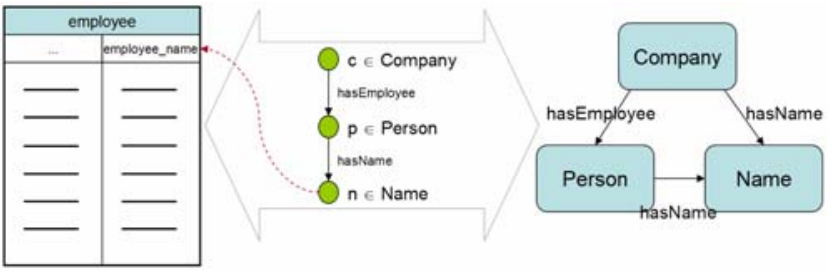

Fig. 1. Annotation as a "bridge" between the resource and the domain ontology

"drawing" relations between them. In addition, the users would benefit from nontrivial "building blocks" provided to them by the software with respect to some Google-like queries.

With this in mind, we have developed Visual OntoBridge [1], a system that provides a graphical user interface and a set of machine learning algorithms that support the user in the annotation task.

\section{Software, Approach, Related Systems}

When the application is launched, the domain ontology is visualized as a graph of concepts. As already said, VOB provides functionality to create several domain ontology instances and interlink them as appropriate to formulate an annotation (see the screenshot in Fig. 2). Establishing annotations manually is not a trivial task, especially if the domain ontology contains a large amount of entities and/or the user is not fully familiar with the conceptualizations in the domain ontology. VOB provides an advanced tool for querying the domain ontology with the purpose of finding the appropriate concepts and triples. The user is able to enter a set of Google-like naturallanguage queries and the system then provides her with two lists of ontology entities: the list of proposed concepts and the list of proposed triples. VOB employs text mining techniques, Page Rank-like algorithms, and consults a Web search engine to populate the two lists of recommended building blocks.

Let us briefly summarize the ontology-querying algorithm. The domain ontology is first represented as a graph in which concepts and triples are represented with vertices and edges represent relations. The user's natural-language query is also represented as a vertex. This is illustrated in Fig. 3. Next, each graph vertex (including the user's query) is assigned a set of documents. The documents are obtained by querying a Web search engine, the search term being either the label of the corresponding ontology entity or the user's query. The set of documents at each particular vertex is converted into TF-IDF vectors and the corresponding centroid vector is computed. The cosine similarity measure is now used to determine similarities between the centroid of the user's query and the rest of the graph. The computed similarity scores are used to weight the edges of the graph. When the graph is properly weighted, PageRank is employed to rank vertices (i.e. concepts and triples) according to the relevance to the query. The vertex representing the query is therefore used as the source vertex for PageRank. Since every concept and every triple has now been ranked by PageRank, it is possible to populate the two required lists of annotation building blocks and present these to the user. Many details and insights can be found in [1]. 


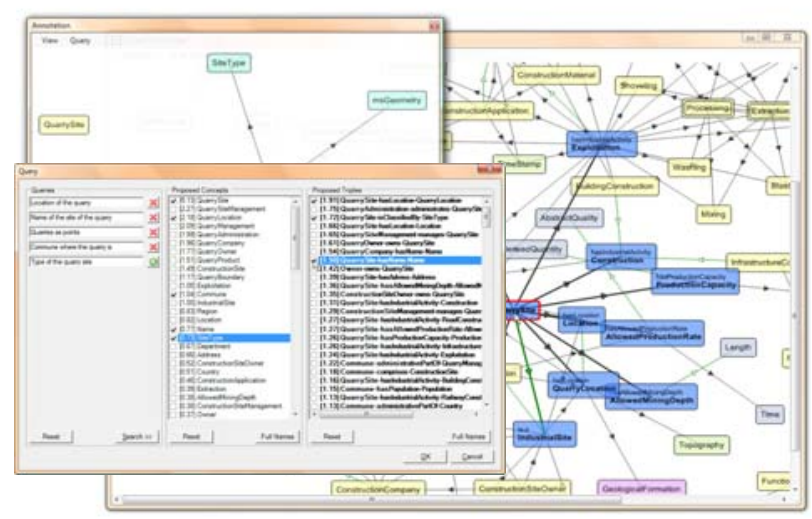

Fig. 2. Visual OntoBridge graphical user interface

Conceptually, our approach can be compared to that of QuestIO [5], a naturallanguage interface to ontologies. The authors of QuestIO argue that related systemssuch as SemSearch, AquaLog, and Orakel-are either keyword-based or require full-blown and correctly phrased questions. Furthermore, such systems are usually domain-specific and require the user to be familiar with the domain. QuestIO does not share these drawbacks. It analyzes a question-written in a natural language-and creates a SeRQL ${ }^{1}$ query from it. Their matching algorithm relies on morphological analysis, POS tagging, and string matching. In contrast to this, our system is not a question-answering system but rather a triple-retrieval system. It does not rely on language analysis algorithms, which makes it suitable for languages that do not yet have adequate support for natural-language processing (e.g. Slovene). Furthermore, our approach is "softer" in the sense that it will always provide answers (i.e. retrieve triples) even if there are no string similarities between query terms and ontology entity labels.

\section{Discussion and Experimental Results}

The presented software was developed and successfully employed in the European project SWING ${ }^{2}$. In SWING, we were annotating geospatial Web services (Web Feature Services or WFS's [3]). More accurately, we were annotating their schemas (i.e. capability documents) to achieve semantic interoperability of services for the purpose of discovery, composition, and execution.

During the project, several WFS were annotated manually (by formulating annotations in the WSML language). For the purpose of evaluating the techniques discussed in Section 0, we asked the domain experts at Bureau of Geological and Mining Research (BRGM, France) to provide us with natural-language queries with which they would hope to retrieve building blocks for these annotations.

\footnotetext{
${ }^{1}$ Sesame RDF Query Language <http://en.wikipedia.org/wiki/RDF_query_language>

${ }^{2}$ Semantic Web Service Interoperability for Geospatial Decision Making <http://www.swingproject.org/>
} 


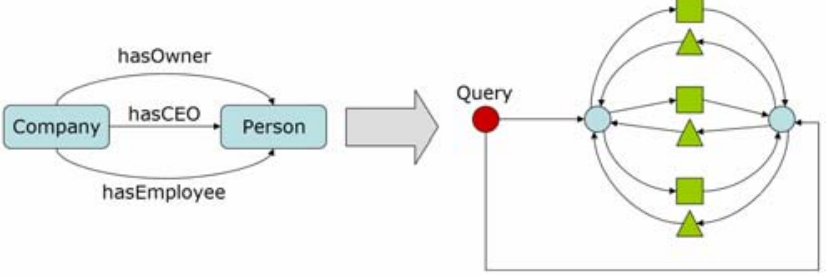

Fig. 3. Representing ontologies as graphs

We measured the Area Under the ROC Curve (AUC) to evaluate the lists of recommendations produced by the algorithm. The rewarding fact is that we managed to significantly beat the baselines which were based solely on term matching (term matching algorithm is described in [4]). We have increased the average AUC for $5.48 \%$ (from $91.46 \%$ to $96.94 \%$ ) on concepts and for $3.18 \%$ on triples (from $93.16 \%$ to $96.34 \%$ ). This presents a big difference from the application point of view. Roughly speaking, the graph-based algorithms are twice as good as the baseline algorithm. Also, the user is able to interact with the system and reformulate queries to achieve even better results. To support this claim, we computed the average AUC by taking, for each annotation, only the most successful annotator into account. The average AUC on the triples rose to $98.15 \%$. The high AUC achieved in the evaluation process is also reflected in practice.

To conclude, VOB incorporates a novel approach to semi-automatic annotation of resources and represents a substantial engineering effort wrapped in an advanced visualization-based graphical user interface.

\section{References}

1. Grcar, M.: D4.5 Software Module for Semantic Annotation of a Web Service. SWING Project Deliverable (2008), http: / /www. swing-project.org/deliverables/document/131

2. Page, L., Brin, S., Motwani, R., Winograd, T.: The PageRank Citation Ranking: Bringing Order to the Web (1999)

3. Open Geospatial Consortium, OGC (2005), Web Feature Service Implementation Specification,

http: //portal.opengeospatial.org/files/?artifact_id=8339

4. Grcar, M., Klien, E., Novak, B.: Using Term-matching Algorithms for the Annotation of Geo-services (extended). In: Post-proceedings of the ECML-PKDD 2007 Workshop on "Web Mining 2.0". Springer, Heidelberg (2007)

5. Tablan, V., Damljanovic, D., Bontcheva, K. In: Proceedings of the 5th European Semantic Web Conference (ESWC 2008), Tenerife, Spain (2008) 